\title{
1 Multiobjective Strain Design: A Framework for Modular Cell Engineering
}

2 Sergio Garcia ${ }^{1,2}$ and Cong T. Trinh ${ }^{1,2^{*}}$

$3 \quad{ }^{1}$ Department of Chemical and Biomolecular Engineering, University of Tennessee, Knoxville, TN

$4 \quad{ }^{2}$ Center for Bioenergy Innovation, Oak Ridge National Laboratory, Oak Ridge, TN

$5 \quad$ *Corresponding author: Tel: 865-974-2181. Email: ctrinh@utk.edu.

6 


\section{ABSTRACT}

9 Diversity of cellular metabolism can be harnessed to produce a large space of molecules. However,

10 development of optimal strains with high product titers, rates, and yields required for industrial

11 production is laborious and expensive. To accelerate the strain engineering process, we have

12 recently introduced a modular cell design concept that enables rapid generation of optimal

13 production strains by systematically assembling a modular cell with an exchangeable production

14 module(s) to produce target molecules efficiently. In this study, we formulated the modular cell

15 design concept as a general multiobjective optimization problem with flexible design objectives

16 derived from mass action. We developed algorithms and an associated software package, named

17 ModCell2 to implement the design. We demonstrated that ModCell2 can systematically identify

18 genetic modifications to design modular cells that can couple with a variety of production modules

19 and exhibit a minimal tradeoff among modularity, performance, and robustness. Analysis of the

20 modular cell designs revealed both intuitive and complex metabolic architectures enabling

21 modular production of these molecules. We envision ModCell2 provides a powerful tool to guide

22 modular cell engineering and sheds light on modular design principles of biological systems.

\section{Keywords}

26 Modular cell engineering; modular cell; production module; modular design; modularity;

27 multiobjective optimization; multiobjective evolutionary algorithm. 


\section{INTRODUCTION}

31 Engineering microbial cells to produce bulk and specialty chemicals from renewable and

32 sustainable feedstocks is becoming a feasible alternative to traditional chemical methods that rely

33 on petroleum feedstocks (1). However, only a handful of chemicals, out of the many possible

34 molecules offered by nature, are industrially produced by microbial conversion, mainly because

35 the current strain engineering process is laborious and expensive for profitable biochemical

36 production (2). Thus, innovative technologies to enable rapid and economical strain engineering

37 are needed to harness a large space of industrially-relevant molecules (3).

The modular organization of biological systems has been a source of inspiration for

39 synthetic biology and metabolic engineering $(4,5)$. Modular pathway engineering breaks down

40 target pathways into tractable pathway modules that can be finely tuned for optimal production of

41 desirable chemicals $(6,7)$. Harnessing combinatorial pathways (e.g., fatty acid biosynthesis,

42 reverse beta oxidation, polyketide or isoprenoid biosynthesis) is one excellent example of modular

43 pathway engineering. These pathways contain metabolic similarity (or combinatorial

44 characteristics) such as a group of common specific enzymes capable of catalyzing linear reaction

45 steps (8) and/or elongation cycles (9-11) and hence are capable of producing a large library of

46 unique molecules (12). Since these molecules are derived from a common precursor metabolite(s),

47 the optimal production strains often share common genotypes and phenotypes, and hence, the

48 costly strain optimization process is only performed once for these molecules. Remarkably, this

49 advantageous strain optimization strategy can be applied even for production of molecules derived

50 from different precursors, using the concept of modular cell (ModCell) design $(2,13,14)$.

51 With the arrival of steady-state, constraint-based stoichiometric models of cellular

52 metabolism, various computational algorithms have been developed to guide strain engineering 
53 (15-17). These methods have featured the design of strains capable of growth-coupled product

54 synthesis $(G C P)$, enabling adaptive laboratory evolution of these designed strains to enhance

55 product titers, rates, and yields $(14,18-20)$. Two approaches on growth-coupled production have

56 been formulated - one based on the coexistence of maximum growth and product synthesis rates

57 during the growth phase (21) and the other based on the obligate requirement of optimal product

58 synthesis in any growth phase (22). The distinction between these two types of growth coupling

59 are also referred to weak coupling $(w G C P)$ and strong coupling $(s G C P)(16,23)$.

60 Development of most strain design algorithms has been focused on overproduction of only

61 one target molecule. The first algorithm proposed for modular cell design compatible for

62 overproduction of multiple target molecules is MODCELL (13), which guided several

63 experimental studies $(14,24-27)$. It works by generating $s G C P$ strain designs for each target

64 product based on elementary mode analysis (28), and then comparing the design strategies of

65 different products to identify common genetic modifications among them. A similar approach was

66 adapted in a subsequent work (29). For MODCELL to find optimal solutions for multiple target

67 products, it requires: 1) enumerating all possible designs above a predefined minimum product

68 yield and with minimal reaction deletion sets for each production network, which might lead to a

69 large number of solutions for each network and hence make the problem computationally

70 intractable, and 2) the resulting designs for all products must be compared to identify common

71 interventions, which is a computationally-hard, set-covering problem. Thus, the current

72 enumerative approach of MODCELL might become intractable very quickly, especially for large-

73 scale metabolic networks and potentially generate non-optimal designs, i.e., requiring more knock-

74 outs than necessary or including fewer products than possible. 
In this study, we generalized the concept of modular cell design and addressed the

76 computational limitation of implementing it. We developed a novel computational platform

77 (ModCell2), based on multiobjective optimization and analysis of mass action of cellular

78 metabolism, to guide the design of modular cells for large-scale metabolic networks. We

79 demonstrated that ModCell2 can systematically identify genetic modifications to design modular

80 cells that can couple with a variety of production modules and exhibit a minimal tradeoff among

81 modularity, performance, and robustness. By analyzing these designs, we further revealed both

82 intuitive and complex metabolic architectures enabling modularity in modular cell and production

83 modules required for efficient biosynthesis of target molecules.

\section{METHODS}

\section{Design principles of modular cell engineering}

87 In the conventional strain engineering approach, a parent strain is genetically modified to yield an

88 optimal production strain to make only a target product. To produce each new molecule, the

89 design-build-test cycles of strain engineering must be repeated, which is laborious and expensive

90 (Figure 1). To minimize the cycles, modular cell engineering is formulated by genetically

91 transforming a parent strain into a modular (chassis) cell that must be assembled with exchangeable

92 modules to create optimal production strains (13). A modular cell is designed to contain core

93 metabolic pathways shared across designed optimal production strains. Exchangeable modules are

94 production pathways designed to synthesize desirable chemicals. A combination of a modular cell

95 and a production module(s) is required to balance redox, energy, and precursor metabolites for

96 sustaining cellular metabolism during growth and/or stationary phases and exhibiting only

97 desirable phenotypes. Practically, modular cell engineering can be applied to monocultures and 
98 polycultures, where a production module(s) can be embedded in a modular cell and activated by

99 intracellular and/or extracellular cues such as light and/or signaling molecules.

101 Multiobjective strain design framework for modular cell engineering

102 For modular cell engineering, we seek to design a chassis cell compatible with as many production

103 modules as possible to achieve only desirable production phenotypes while requiring minimal

104 genetic modifications. Since all production modules must leverage cellular resources of the

105 modular cell (e.g. precursor metabolites, cofactors, and energy), they form competing objectives.

106 Therefore, the framework of modular cell engineering can be formulated as a multiobjective

107 optimization problem, named ModCell2, as described below.

$108 \operatorname{maximize}_{\text {over } y_{j}, z_{j k}} \boldsymbol{F}=\left(f_{1}, f_{2}, \ldots, f_{|K|}\right)^{T}$

109 subject to

$110 \quad f_{k} \in \arg \max \left\{\sum_{j \in J_{k}} c_{j k} v_{j k}\right.$

$111 \quad$ subject to

$112 \quad \sum_{j \in J_{k}} S_{i j k} v_{j k}=0$, for all $i \in I_{k}$

113

$$
l_{j k} \leq v_{j k} \leq u_{j k}, \text { for all } j \in J_{k}
$$

$$
l_{j k} d_{j k} \leq v_{j k} \leq u_{j k} d_{j k}, \text { for all } j \in C \text { and } C \subseteq J_{k}
$$

115

$$
\text { where } \left.d_{j k}=y_{j} V z_{j k}\right\}, \text { for all } k \in K
$$

$$
z_{j k} \leq\left(1-y_{j}\right), \text { for all } j \in C, k \in K
$$

$117 \quad \sum_{j \in C}\left(1-y_{j}\right) \leq \alpha$

118

$$
\sum_{j \in C} z_{j k} \leq \beta_{k}, \text { for all } k \epsilon K
$$


119 where $i, j$, and $k$ are indices of metabolite $i$, reaction $j$, and production network $k$, respectively; $f_{k}$

120 is a design objective for network $k$; $c_{j k}$ represents the cellular objective for reaction $j$ in network $k$

121 associated with a design objective defined in eqns. 8-10; $v_{j k}(\mathrm{mmol} / \mathrm{g} \mathrm{DCW} / \mathrm{h})$ is metabolic flux of

122 reaction $j$ bounded by $l_{j k}$ and $u_{j k}$ in network $k$, respectively; $y_{j}$ and $z_{j k}$ are binary design variables

123 for deletion reaction $j$ and module reaction $j$ in network $k$, respectively; $\alpha$ and $\beta_{k}$ are design

124 parameters for deletion and module reactions, respectively; $S_{i j k}$ is a stoichiometric coefficient of

125 metabolite in reaction $\mathrm{j}$ of network $k$; and $C$ (eqn. 4) is the candidate reaction set (Supplementary

126 File S1). The goal of the optimization problem is to simultaneously maximize all design objectives

$127 f_{k}$.

128 Steady-state mass balance constraint of cellular metabolism. Quasi steady-state flux

129 balance of cellular metabolism (eqn. 2) is used as metabolic constraints for eqn. 1 (30, 31). A

130 model corresponding to each modular production strain (i.e. production network k) will be derived

131 from a parent strain (i.e. parent network) by adding necessary reactions (e.g., a production module)

132 to produce a target molecule. A feasible flux distribution for each production network is described

133 by mass balance (eqn. 2) and reaction flux bounds (eqns. 3 and 4). For a given production network,

134 the phenotypic space can be illustrated by the gray area that is projected onto the two-dimensional

135 space spanned by product synthesis and growth rates (Figure 2).

136 Design variables. In our formulation for modular cell engineering, we introduced two

137 design variables - binary reaction deletions $\left(y_{j}\right)$ inherent to the modular cell and module-specific

138 reaction insertions $\left(z_{j k}\right)$ (eqn. 4). These variables can be experimentally manipulated to constrain

139 the desirable phenotypes of production strains as shown in Figure 2. Specifically, $y_{j}=0$ if reaction

$140 j$ is deleted from the modular cell; otherwise, $y_{j}=1$. Deleting metabolic reactions removes

141 undesired functional states of the network and leaves those with high design objectives. Likewise, 
$142 z_{j k}=1$ if reaction $j$ is present in the production network $k$; otherwise, $z_{j k}=0$. These module reactions

143 are endogenous reactions removed from the parent network (eqn. 5), but are added back to a

144 specific production module to enhance the compatibility of a modular cell. The maximum number

145 of reaction deletions $(\alpha)$ and module-specific reaction insertions $\left(\beta_{k}\right)$ are user-defined parameters.

146 Design objectives. To generalize ModCell2 design, we allow three different types of

147 design objectives $\left(f_{k}\right.$, eqn. 1) that determine production phenotypes for each production network.

148 Depending on the application, a phenotype can be designed to be weak coupling ( $w G C P$ ), strong

149 coupling $(s G C P)$, and/or non-growth production $(N G P)$ (Figure 2). The constrained phenotypic

150 spaces based on these design objectives are shown in color; any point within these spaces is a

151 feasible physiological state of the cell that can be represented by a metabolic flux distribution.

152 The $w G C P$ design seeks to achieve a high product rate at maximum growth rate (Figure

153 2A). The $w G C P$ design objective, $f_{k}^{w G C P}(\in[0,1])$, is calculated as follows:

$$
f_{k}^{w G C P}=\frac{v_{P k}^{\mu}}{v_{P \operatorname{maxk}}^{\mu}}
$$

155 where $v_{P k}^{\mu}$ is the minimum synthesis rate of the target product $\mathrm{P}$ at the maximum growth rate for

156 production network $k$ and $v_{P \operatorname{maxk}}^{\mu}$ is the maximum synthesis rate of $\mathrm{P}$ (Supplementary File S1).

157 This $w G C P$ design formulation is equivalent to RobustKnock (32) or OptKnock with a tilted

158 objective function $(21,33,34)$. In eqn. $8, f_{k}^{w G C P}$ is scaled from 0 to 1 for proper comparison among

159 products. The $w G C P$ design is appropriate for applications where growth rate is not limited by the

160 nutrients, and the product is formed during the growth phase.

161 The $s G C P$ design seeks to achieve a high product rate not only at optimal growth rate but

162 also during non-growth phase (Figure $2 \mathrm{~B})$. The $s G C P$ design objective, $f_{k}^{s G C P}(\in[0,1])$, is

163 calculated as follows: 


$$
f_{k}^{S G C P}=\frac{v_{P k}^{\mu}}{v_{P_{\text {max }}}^{\mu}} \cdot \frac{v_{P k}^{\bar{\mu}}}{v_{P_{\text {max }}}^{\bar{\mu}}}
$$

165 where $v_{P k}^{\bar{\mu}}$ and $v_{P_{m a x}}^{\bar{\mu}}$ are the minimum and maximum product formation rates for production

166 network $k$ in the stationary phase, respectively (Supplementary File S1). The $s G C P$ design

167 objective is comparable to the one implemented in MODCELL (13). Different from $w G C P, s G C P$

168 requires high product synthesis rate for any growth phase. However, the additional constraint of

169 optimal product synthesis during the stationary phase requires more genetic manipulations or

170 specific experimental conditions (e.g., anaerobic growth condition, supply of intermediate

171 metabolites). Both $w G C P$ and $s G C P$ designs enable fast growth selection to attain the optimum

172 product rates by adaptive laboratory evolution $(35,36)$.

173 The NGP design aims to maximize the minimum product rate during the non-growth phase

174 by eliminating carbon fluxes directed to biomass synthesis (Figure $2 \mathrm{C}$ ). The $N G P$ design objective,

$175 f_{k}^{N G P}(\in[0,1])$, is calculated as follows:

$$
f_{k}^{N G P}=\frac{v_{P k}^{\bar{\mu}}}{v_{P \max }^{\bar{\mu}}}
$$

177 While the NGP design is not suitable for growth selection, it can be derived from a $w G C P$ (or

$178 s G C P$ ) design by imposing additional genetic modifications. Practically, NGP design strains can

179 be activated during cell culturing using a regulatory genetic circuit to toggle switch between

180 production phases.

181 Design solutions. Optimal solutions for eqn. 1 are a Pareto set (PS) that correspond to

182 design variables, including reaction deletions $\left(y_{j}\right)$ and module reaction insertions $\left(z_{j k}\right)$. Each

183 solution constitutes a design of a modular cell:

$$
P S:=\{\mathbf{x} \in \Omega: \nexists \mathbf{t} \in \mathbf{\Omega}, \mathbf{F}(\mathbf{t}) \prec \mathbf{F}(\mathbf{x})\}
$$


185 Here, $\mathbf{F}(\mathbf{t}) \prec \mathbf{F}(\mathbf{x})$ means $\mathbf{F}(\mathbf{t})$ dominates $\mathbf{F}(\mathbf{x})$ if and only if $\mathrm{f}_{\mathrm{i}}(\mathbf{t}) \geq \mathrm{f}_{\mathrm{i}}(\mathbf{x})$ for all $i$, and $\mathbf{F}(\mathbf{t})$ differs

186 from $\mathbf{F}(\mathbf{x})$ in at least one entry. The feasible space of design variables, $\Omega$, is defined by the problem

187 constraints (eqn. 2-7, also see Supplementary File S1). Phenotypes of modular cells will be the

188 image of the Pareto set in the objective space, known as the Pareto front $(\boldsymbol{P F})$ :

$$
\boldsymbol{P F}:=\{\mathbf{F}(\mathbf{x}): \mathbf{x} \in \boldsymbol{P S}\}
$$

190 For the multiobjective strain design framework, the input parameters include $\alpha$ (eqn. 6), $\beta_{k}$ (eqn.

191 7), and the production networks as input metabolic models. Each model contains a production

192 module to produce one target chemical. The output is a Pareto set (genetic modifications) and its

193 respective Pareto front (desirable production phenotypes). For a special case with no trade-off

194 among the design objectives, an optimal solution, named a utopia point, exists where each

195 objective achieves its maximum value. The multiobjective strain design formulation presented is

196 general and can be applied to design modular cells for any organism.

\section{Algorithm and implementation}

ModCell2 algorithm. To solve the multiobjective optimization problem for modular cell engineering, we used multiobjective evolutionary algorithms (MOEAs) (37). MOEAs were

201 selected because they can efficiently handle linear and non-linear problems and do not require

202 preferential specification of design objectives (38). MOEAs start by randomly generating a 203 population of individuals (a vector of design variables), each of which is mapped to a design 204 objective vector (i.e., a fitness vector). In ModCell2 (Supplementary File S1), the objective values 205 of an individual are calculated by solving the linear programming problems for each production 206 network. Next, individuals are shuffled to generate an offspring, from which the most fit 
individuals are kept. This process was repeated until the termination criteria was reached, for instance, either the solutions cannot be further improved or the simulation time limit is reached.

210 ModCell2 software package based on three core classes (Figure S1 in Supplementary File S2).

211 The Prodnet class parses and pre-processes production network models, and computes production

212 phenotypes. The MCdesign class serves as an interface between the MOEA optimization method 213 and metabolic models. Finally, the ResAnalysis class loads the Pareto set computed by MCdesign

214 and identifies the most promising modular cell designs.

215 The code was written in MATLAB 2017b (The Mathworks Inc.) using the function

216 gamultiobj() from the MATLAB Optimization Toolbox that implements the NSGA-II algorithm

217 (39) to solve the multiobjective optimization problem. The solution and analysis methods were

218 parallelized using the MATLAB Parallel Computing Toolbox. The linear programs to calculate

219 metabolic fluxes were solved using the GNU Linear Programming Kit (GLPK). The COBRA

220 toolbox $(40,41)$ and F2C2 $0.95 b(42)$ were also used for COBRA model preprocessing and

221 manipulation.

222 Metabolic models. In our study, we used three parent models including i) a small metabolic

223 network to illustrate the modular cell design concept (13), ii) a core metabolic network of

224 Escherichia coli to compare the performance of ModCell2 with respect to the conventional single-

225 product strain design strategy and the first-generation modular cell design platform MODCELL

226 (13), and iii) a genome-scale metabolic network of E. coli (i.e., iML1515 (33)) for biosynthesis of

227 a library of endogenous and heterologous metabolites, including 4 organic acids, 6 alcohols, and

22810 esters (Figures S2 in Supplementary File S2) (8, 22, 25, 43-49). 
Simulation protocols. Anaerobic conditions were imposed by setting oxygen exchange

fluxes to be 0 , and the glucose uptake rate was constrained to be at most $10 \mathrm{mmol} / \mathrm{gCDW} / \mathrm{h}$, as

231 experimentally observed for $E$. coli. When using the genome-scale model iML1515 to simulate

$232 w G C P$ designs, the commonly observed fermentative products (acetate, $\mathrm{CO} 2$, ethanol, formate,

233 lactate, succinate) were allowed for secretion as described elsewhere (50). For simulation of $s G C P$

234 and $N G P$ designs, the glucose uptake rate was fixed (i.e., $-10 \mathrm{mmol} / \mathrm{gCDW} / \mathrm{h}$ ); otherwise, the flux

235 is not active during the no-growth phases, resulting in the product synthesis rate of 0 regardless of

236 genetic manipulations. To compare ModCell2 with Optknock, we applied the OptKnock algorithm

237 with a titlted objective function (51) to generate $w G C P$ designs for each production network, using

238 the open-source algebraic modeling language Pyomo (52). The MILP problems were solved using

239 CPLEX 12.8.0 with a time limit of 10,000 seconds set for each product. ModCell2 is provided as

240 an open-source software package and is freely available for academic research. The software

241 package and documentation can be download via either web.utk.edu/ ctrinh or Github

242 https://github.com/TrinhLab.

\section{Analysis methods for design solutions}

245 Compatibility. The compatibility, $C\left(€ Z^{+}\right)$, of a design is defined as the number of products

246 that are coupled with a modular cell and has objective values above a specified cutoff value $\theta$. As

247 a default, we set $\theta=0.6$ for the $w G C P$ and $N G P$ design objectives and $\theta=0.36\left(0.6^{2}\right)$ for the $s G C P$

248 design objective. For example, a $w G C P$ design for 3 products that has the design objective values

249 of $0.4,0.9$, and 0.6 has a compatibility of 2 , given a cutoff value of $\theta \geq 0.6$.

250 Compatibility difference and loss. Robustness is the ability of a system to maintain its

251 function against perturbations, and hence is very important of designing biological and engineered 
systems (53). To evaluate the robustness of modular cell designs, we defined two metrics, the compatibility difference $(C D)$ and compatibility loss $(\mathrm{CL} \in[0,1])$ as follows:

$$
\begin{aligned}
& C D=C_{\text {initial }}-C_{\text {final }} \\
& C L=\frac{C_{\text {initial }}-C_{\text {final }}}{C_{\text {initial }}}
\end{aligned}
$$

where $C_{\text {initial }}$ and $C_{\text {final }}$ are the compatibilities of a modular cell design before and after a single

257 reaction deletion, respectively. The value $C D>0$ (or $C L>0$ ) means the modular gains fitness

258 while $C D<0$ (or $C L<0$ ) means that it loses its fitness. In the analysis, we did not consider essential

259 and blocked reactions for our single-deletion analysis; for instance, there are only 1139 potential

260 reaction deletions in the iML1515 model.

Metabolic switch design. A metabolic switch design is a modular cell that can possess

262 multiple production phenotypes (i.e., $w G C P, s G C P$, and $N P G$ ), activated by an environmental

263 stimulus (e.g. metabolites, lights). The metabolic switch design is enforced to have a set of reaction

264 (gene) deletions in one production phenotype to be a subset of the other, for instance, $\left\{\mathbf{y}_{\mathrm{wGCP}}\right\} \subseteq$

$265\left\{\mathbf{y}_{\mathrm{NPG}}\right\},\left\{\mathbf{y}_{\mathrm{wWCP}}\right\} \subseteq\left\{\mathbf{y}_{\mathrm{sGCP}}\right\}$. The metabolic switch design is beneficial for multiphase fermentation

266 configurations that enable flexible genetic modification and implementation. Specifically, the

267 metabolic switch design can exhibit the ${ }^{2} G C P$ phenotype during the growth phase and the $N P G$

268 (or $s G C P$ ) phenotype during the stationary phase. The metabolic switches can be implemented

269 using the genetic switchboard (54).

271 RESULTS AND DISCUSSION

272 Illustrating ModCell2 for modular cell design of a simplified network

273 An example parent network, adapted from (13), was used to illustrate ModCell2 (Figure 3A).

274 Inputs for the multiobjective optimization problem include i) three production networks (Figure 
$2753 \mathrm{~B}$ ), comprising of one endogenous production module (module 1) and two heterologous

276 production modules (modules 2 and 3) and ii) design parameters (Figure 3C), containing design

277 objective type, maximum number of deletion reactions $(\alpha)$, and maximum number of module

278 reactions $\left(\beta_{k}\right)$. The output of ModCell2 generated the Pareto set and the corresponding Pareto front

279 for modular cell designs (Figure 3D). The 2-D plots of product yields versus growth rates presented

280 the feasible phenotypic spaces of the wildtype (gray area) and the designed strain (blue area).

283 (Figure 3D). For instance, by setting $\alpha=3$ and $\beta_{k}=0$, we found three $s G C P$ designs including

$284 s G C P-3-0-1, s G C P-3-0-2$, and $s G C P-3-0-3$. The first design $s G C P-3-0-1$ has a compatibility of 2

285 (given $\theta>0$ ) with the design objective values of 0.42 and 0.97 for the products $\mathrm{P} 2$ and P3,

286 respectively. In contrast, the $s G C P-3-0-2$ and $s G C P-3-0-3$ designs have compatibilities of only 1

287 with the design objectives of 0.63 for $\mathrm{P} 2$ and 0.45 for $\mathrm{P} 1$, respectively.

288 Based on all designs, we can clearly see the trade-offs for optimization of different products

289 for $\beta_{k}=0$. However, setting $\beta_{k} \geq 1$ helps increase the compatibility of a modular cell with

290 different production modules. In addition, we found that the Pareto front collapses into a utopia

291 point as seen in the $w G C P-1-1-1, s G C P-3-1-1$, and $N G P-3-1-1$ designs. For instance, the modular

292 cell, $s G C P-3-1-1$, is compatible with all three products. The three corresponding optimal

293 production strains can couple growth and product formation during the growth phase. During the

294 stationary phase, these strains produce the products at maximum theoretical yields. In theory, a

295 universal modular cell always exists, provided that enough reaction deletions and module reactions

296 are used. It might be more tractable to construct such a modular cell from a synthetic minimal cell

297 using the bottom-up approach. However, construction of a universal modular cell from a host 
organism (e.g., E. coli, S. cerevisiae) using the top-down approach will require a significantly large number of genetic modifications, that might be challenging.

Comparing ModCell2 designs with first-generation MODCELL and single product designs ModCell2 can generate more and better designs than the first-generation modular cell

In the first case study, we fixed the reaction module, i.e. $\beta_{k}=2$ for ethanol dehydrogenase

311 with the same $s G C P$ objective values like MODCELL (Figure 4B, 4C, 4D) together with other

312 alternative solutions (Supplementary File S3). Interestingly, ModCell2 only required 5 and 6

313 reaction deletions as opposed to 7 and 7 for the $s G C P-5-0-5$ and $s G C P-5-0-6$ designs, respectively.

314 By setting the maximum reaction deletions to $\alpha \geq 6$, ModCell 2 could find better design solutions

315 with fewer deletion reaction requirement and higher objective values (Supplementary File S3).

317 module reactions. By setting $\alpha=5$ and $\beta_{k}=1$, we found the $s G C P-5-1-8$ design that is

318 compatible with all products and achieves the same objective values for products found in $s G C P$ -

$3195-0-5, s G C P-5-0-6$, and $s G C P-5-0-2$ (Figure 4E). The desirable phenotypic spaces can be further

320 constrained for many products if $\alpha$ is increased from 5 to 6 (Figure 4F). Remarkably, by setting 
$321 \alpha=8$ and $\beta_{k}=2$, we found a utopia point design, $s G C P-8-2-9$, without any trade-off among

322 design objectives (Figure 4G). This utopia point design could not be achieved with $\alpha<8$

323 regardless of any $\beta_{k}$ value.

324 Overall, the results demonstrate that ModCell2 can efficiently compute the Pareto front of

325 modular cell designs. It can find better designs with fewer reaction deletion and module reaction

326 requirements, improve design objective values, and enhance compatibility.

328 product designs. To evaluate if the conventional, single-product design strategy is suitable for

329 modular cell engineering, we first used OptKnock to generate $w G C P$ designs for the same 10 target

330 molecules independently with various allowable reaction deletions $(\alpha=2,3, \ldots, 7)$. Likewise, we

331 employed ModCell2 to produce $w G C P$ designs using the same $\alpha$ and various $\beta$. To directly

332 compare OptKnock and ModCell2 solutions, we calculated the $w G C P$ design objective values for

333 all products based on each OptKnock solution (Supplementary File S3). As expected, our result

334 showed that ModCell2 and OptKnock designs have the same highest objective values for each

335 product (Figure 5A). However, several OptKnock solutions were always dominated by ModCell2

336 solutions in all parameter configurations (Figure 5B). With $\alpha \geq 4$, ModCell2 could identify

$337 w G C P-\alpha-1$ designs with the maximum compatibility of 10, while the best OptKnock designs only

338 achieved the highest compatibility of 5 (Figure 5C, 5D).

339 Overall, ModCell2 can generate modular cells compatible with the maximum number of

340 modules and achieve high objective values. Single-product designs might not be compatible with

341 a large number of products, and the solutions might be far from Pareto optimality. 


\section{Exploring emergent features of modular cell design using an $E$. coli genome-scale network}

346 generate $w G C P$ designs for 20 target molecules with $\alpha=4$ and various $\beta_{k}$ (Supplementary File

347 S4). The $\alpha$ value was chosen because with 4 deletions, OptKnock could identify single product

348 designs with objectives above $60 \%$ of the theoretical maximum (Supplementary File S5). With

$349 \beta_{k}=0$, ModCell2 could identify modular cell designs with compatibility of 17 , for example, the

$350 w G C P-4-0-50$ design featuring deletion of ACALD (acetaldehyde dehydrogenase, adhE),

351 ACKr/PTAr (acetate kinase, ack; phosphotransacetylase, pta), GLYAT (glycine C-

352 acetyltransferase, $k b l$ ), and LDH_D (lactate dehydrogenase, $l d h A$ ) (Figure 6A, 6D, Supplementary

353 File S4). By analyzing all $w G C P-4-\beta_{k}-d$ designs (257 total for $\beta_{k}=0,1,2$, and 3), we found that

354 the ethanol and D-lactate production modules are most compatible with all modular cell designs

355 (Figure 6A, 6C, Supplementary File S4). Among reaction deletions, LdhA (86\% of designs), Pta

356 (38\%), and $\operatorname{AdhE}(25 \%)$ are the most frequent deletion reactions (Figure 6B). This finding is

357 consistent with a comprehensive survey of metabolic engineering publications (55) showing that

358 these deleted reactions appeared in most of E. coli engineered strains for production of fuels and

359 chemicals. The result supports the potential use of modular cell engineering to systematically build 360 modular platform strains.

362 evaluate whether ModCell2 could capture the combinatorial properties among production

363 modules, we analyzed the Pareto front of $w G C P-4-0-d$ that have a total of 58 designs. Hierarchical

364 clustering of this Pareto front revealed certain products with similar objective values across

365 solutions, such as ethyl esters and butyrate esters (Figure 6A). These products together were 
compatible with different modular cells and exhibited metabolic similarity in their production

modules. Thus, ModCell2 could generate designs that capture the combinatorial properties useful

368 for modular cell engineering.

ModCell2 can identify highly compatible modular cells. Analysis of compatibility shows

370 that certain modular cells can couple with production modules that may not exhibit the

371 combinatorial properties (Figure 6D). However, there exists a tradeoff between the number of

372 feasible designs and degree of compatibility. Some modular cell designs are compatible with up

373 to 17 out of 20 products, for instance, the most compatible design, $w G C P-4-0-48$, featuring

374 deletions of ACALD (adhE), ACKr/PTAr (ack, pta), GND (phosphogluconate dehydrogenase,

375 gnd ) and LDH_D (ldhA) (Supplementary File S4). An alternative design wGCP-4-0-48-alternative

376 also exists where deletion of G6PDH2r (gluose-6-phosphate dehydrogenase, $z w f$ ) is replaced by

377 that of GND, the first step in the oxidative pentose phosphate pathway. The gene deletions in the

378 design wGCP-4-0-48-alternative are a subset of the modular E. coli strain TCS095, whose modular

379 properties have recently been validated experimentally (14).

To determine if modular cell design is a viable alternative to single-product design, we also

381 analyzed a potential tradeoff between design performance and modularity by comparing the

382 maximum value of each objective across all solutions in the Pareto front and the single-product

383 design optima. If production modules exhibit competing phenotypes, a modular cell will not

384 achieve the same performance in all modules as a single-product design strain. Analysis of the

385 most compatible design wGCP-4-0-48-alternative showed that it could achieve objectives within

$3864 \%$ of the single-product optima in 14 products and within $10 \%$ in 3 products (Figure $6 \mathrm{E}$ ). This

387 result indicates that it is feasible to identify highly compatible modular cell designs without a

388 significant tradeoff between performance and modularity. 
391 change $(C D)$ of $w G C P-4-0$ designs with compatibilities of 4 or greater (Figure S3 in

392 Supplementary File 2). Remarkably, the result shows that only $7.5 \%$ of potential reaction deletions

393 were detrimental to the robustness of modular cells while the large remaining portion did not affect

$394 C D$ values. Out of the 85 reactions whose deletion affected compatibility, only a few appeared 395 consistently across the designs. For instance, deletion of TPI (triose-phosphate isomerase, tpi) led

396 to an average compatibility loss of $95 \%$, inactivating most modular cell designs. Based on flux

397 variability analysis, TPI must operate in the forward direction by converting glycerone phosphate

398 (dhap) to glyceraldehyde-3-phosphate (g3p) to drive sufficient flux through glycolysis and hence

399 preventing synthesis of undesired byproducts (D-lactate or 1,2-propanediol) from dhap. Likewise,

400 deletion of carbon dioxide and water transport and exchange reactions caused compatibility loss

401 across all designs. Pyruvate carboxylase (PPC) is an important reaction to channel carbon flux 402 through the Krebs cycle (56), and hence, deletion of PPC reduces compatibility in most modular 403 cell designs with an average $C L$ of $43 \%$.

404 While some reaction deletions are critical for modular cell robustness, others are associated 405 with specific products. For example, deletion of PDH (pyruvate dehydrogenase complex, 406 lpd/aceEF) removes compatibility in all butanol-derived designs, indicating PFL (pyruvate 407 formate lyase, $p f l$ ) is not an appropriate route. To make heterologous butanol-derived molecules 408 under anaerobic conditions, FDH (NADH-dependent formate dehydrogenase, $f d h$ ) is required in 409 butanol-derived modules where enzymatic reaction pairs of PFL and FDH could substitute PDH 410 known to be anaerobically inhibited. 
Overall, analysis of tradeoff between modularity and robustness can identify not only the

412 conserved metabolic features of modular cells but also potential bottlenecks in specific production

413 modules.

414

Enabling metabolic switch among different design objectives using ModCell2

The ability to dynamically control growth and production phases can potentially enhance

417 product titers, rates, and yields. For instance, two-phase fermentation can be employed where

418 growth phase is optimized for biomass synthesis and stationary phase for chemical production

419 (57). Using ModCell2, we investigated the feasibility to design optimal strains to toggle switch

420 desirable production phenotypes.

421 To design a $w G C P \rightarrow N P G$ metabolic switch, we first used our reference $w G C P$ design as

422 a parent strain (Figure 7A) and then employed ModCell2 to identify the most compatible

$423 w G C P \rightarrow N P G$ designs. With 5 additional deletions, we could find $w G C P \rightarrow \mathrm{NPG}$ designs that

424 encompass both $w G C P$ and $N P G$ phenotypes, for instance, the sup-NGP-5-0-23 design featuring

425 deletion of PGI (glucose-6-phosphate isomerase, pgi), MDH (malate dehydrogenase, $m d h$ ), ASPT

426 (L-aspartase, aspT), Tkt2 (transketolase, $t k t B$ ), and ATPS4rpp (ATP synthase, atp) (Figure 7B).

427 The deletion reactions in the $w G C P \rightarrow$ NPG designs appear in both catabolic (PGI, ATPS4tpp) and

428 anabolic (ASP, TKT2) processes, responsible for growth disruption and direction of carbon flow

429 to the biosynthesis of target products.

430 Likewise, we used ModCell2 to design a $w G C P \rightarrow s G C P$ metabolic switch. We identified

431 the most compatible $w G C P \rightarrow s G C P$ designs with 6 additional deletions, for instance, the sup-

$432 s G C P-6-0-39$ design featuring the deletion of MGSA (Methylglyoxal synthase, $m g s A$ ), ALCD2x

433 (alcohol dehydrogenase, $a d h E$ ), PFL, MDH, FADRx (FAD reductase, fadI), and GLUDy 
$434\left(\mathrm{NADP}^{+}\right.$dependent glutamate dehydrogenase, $g d h A$ ) (Figure $7 \mathrm{C}$ ). Different from the $w G C P \rightarrow N G P$

435 metabolic switch, all deletions in the $w G C P \rightarrow s G C P$ designs are involved the elimination of

436 biosynthesis pathways of undesirable byproducts.

437 While it is feasible to metabolically switch among different production phenotypes, it not 438 only requires more reaction deletions but also reduces the product compatibility. For instance, the $439 w G C P \rightarrow \mathrm{sGCP}$ and $w G C P \rightarrow \mathrm{NGP}$ designs are only compatible with 5 products while the $w G C P$ 440 parent design have a compatibility of 17 out of 20 products with 4 deletions. The main reason is 441 that both $w G C P \rightarrow \mathrm{sGCP}$ and $w G C P \rightarrow \mathrm{NGP}$ designs must eliminate all possible redundant 442 pathways that result in biosynthesis of undesirable byproducts.

\section{CONCLUSION}

445 In this study, we developed a multiobjective strain design platform for modular cell 446 engineering. With a new developed algorithm and computational platform, ModCell2 enables

447 flexible design of modular cells that can couple with production modules to exhibit desirable 448 production phenotypes. In comparison to the first-generation strain design platform, ModCell2 can

449 handle large-scale metabolic networks and identify better solutions that require fewer genetic 450 modifications and exhibit more product compatibility. Different from the conventional single451 product strain design, ModCell2 can find solutions that are Pareto optimal with negligible tradeoffs 452 among modularity, performance, and robustness. We envision ModCell2 is a useful tool to 453 implement modular cell engineering and fundamentally study modular designs in natural and 454 synthetic biological systems. 


\section{ACKNOWLEDGEMENTS}

458 This research was financially supported in part by the NSF CAREER Award

459 (NSF\#1553250) and the DOE subcontract Grant (DE-AC05-000R22725) by the Center of

460 Bioenergy Innovation (CBI), the U.S. Department of Energy Bioenergy Research Center funded

461 by the Office of Biological and Environmental Research in the DOE Office of Science. The funders

462 had no role in the study design, data collection and analysis, decision to publish, or preparation of

463 the manuscript.

464 


\section{REFERENCES}

466 1. Nielsen J \& Keasling Jay D (2016) Engineering Cellular Metabolism. Cell 164:1185-1197.

467 2. Trinh CT \& Mendoza B (2016) Modular cell design for rapid, efficient strain engineering $468 \quad$ toward industrialization of biology. Current Opinion in Chemical Engineering 14:18-25.

469 3. Connelly $\mathrm{T}$, et al. (2015) Industrialization of Biology: A Roadmap to Accelerate the $470 \quad$ Advanced Manufacturing of Chemicals.

471 4. Sauro HM (2008) Modularity defined. Molecular systems biology 4.

472 5. Purnick PEM \& Weiss R (2009) The second wave of synthetic biology: from modules to $473 \quad$ systems. Nature reviews Molecular cell biology 10:410-422.

474 6. Yadav VG, De Mey M, Giaw Lim C, Kumaran Ajikumar P, \& Stephanopoulos G (2012)

475 The future of metabolic engineering and synthetic biology: Towards a systematic practice.

$476 \quad$ Metabolic Engineering 14:233-241.

477 7. Biggs BW, De Paepe B, Santos CNS, De Mey M, \& Ajikumar PK (2014) Multivariate 478 modular metabolic engineering for pathway and strain optimization. Current opinion in 479 biotechnology 29:156-162.

480 8. Rodriguez GM, Tashiro Y, \& Atsumi S (2014) Expanding ester biosynthesis in Escherichia 481 coli. Nat Chem Biol 10:259-265.

482 9. Cheong S, Clomburg JM, \& Gonzalez R (2016) Energy-and carbon-efficient synthesis of 483 functionalized small molecules in bacteria using non-decarboxylative Claisen $484 \quad$ condensation reactions. Nature biotechnology 34:556-561.

485 10. Tseng H-C \& Prather KL (2012) Controlled biosynthesis of odd-chain fuels and chemicals 486 via engineered modular metabolic pathways. Proceedings of the National Academy of Sciences 109(44):17925-17930. 
488 11. Xu P, et al. (2013) Modular optimization of multi-gene pathways for fatty acids production 489 in E. coli. Nat Commun 4:1409.

490 12. Ng CY, Chowdhury A, \& Maranas CD (2016) A microbial factory for diverse chemicals. $491 \quad$ Nat Biotech 34:513-515.

492 13. Trinh CT, Liu Y, \& Conner DJ (2015) Rational design of efficient modular cells. Metabolic $493 \quad$ engineering 32:220-231.

494 14. Wilbanks B, Layton D, Garcia S, \& Trinh C (2017) A Prototype for Modular Cell 495 Engineering. ACS Synthetic Biology:acssynbio.7b00269.

496 15. Long MR, Ong WK, \& Reed JL (2015) Computational methods in metabolic engineering 497 for strain design. Current opinion in biotechnology 34:135-141.

498 16. Machado D \& Herrgård MJ (2015) Co-evolution of strain design methods based on flux 499 balance and elementary mode analysis. Metabolic Engineering Communications 2:85-92.

500 17. Chowdhury A, Zomorrodi AR, \& Maranas CD (2015) Bilevel optimization techniques in 501 computational strain design. Computers \& Chemical Engineering 72:363-372.

502 18. Fong SS, et al. (2005) In silico design and adaptive evolution of Escherichia coli for 503 production of lactic acid. Biotechnology and Bioengineering 91:643-648.

504 19. Trinh CT \& Srienc F (2009) Metabolic engineering of Escherichia coli for efficient 505 conversion of glycerol to ethanol. Applied and Environmental Microbiology 75(21):6696$506 \quad 6705$.

507 20. Otero JM, et al. (2013) Industrial systems biology of Saccharomyces cerevisiae enables $508 \quad$ novel succinic acid cell factory. PLoS One 8:e54144. 
509 21. Burgard AP, Pharkya P, \& Maranas CD (2003) Optknock: a bilevel programming

510 framework for identifying gene knockout strategies for microbial strain optimization.

511 Biotechnology and Bioengineering 84:647-657.

512 22. Trinh CT, Unrean P, \& Srienc F (2008) Minimal Escherichia coli Cell for the Most

513 Efficient Production of Ethanol from Hexoses and Pentoses. Applied and Environmental

$514 \quad$ Microbiology 74(12):3634-3643.

515 23. Klamt S \& Mahadevan R (2015) On the feasibility of growth-coupled product synthesis in 516 microbial strains. Metabolic engineering 30:166-178.

517 24. Wierzbicki M, Niraula N, Yarrabothula A, Layton DS, \& Trinh CT (2016) Engineering an 518 Escherichia coli platform to synthesize designer biodiesels. Journal of biotechnology $519 \quad 224: 27-34$

520 25. Layton DS \& Trinh CT (2014) Engineering modular ester fermentative pathways in $521 \quad$ Escherichia coli. Metabolic Engineering 26:77-88.

522 26. Layton DS \& Trinh CT (2016) Expanding the modular ester fermentative pathways for 523 combinatorial biosynthesis of esters from volatile organic acids. Biotechnology and $524 \quad$ bioengineering.

525 27. Layton DS \& Trinh CT (2016) Microbial synthesis of a branched-chain ester platform from $526 \quad$ organic waste carboxylates. Metabolic Engineering Communications 3:245-251.

527 28. Trinh CT, Wlaschin A, \& Srienc F (2009) Elementary mode analysis: a useful metabolic 528 pathway analysis tool for characterizing cellular metabolism. Applied Microbiology and $529 \quad$ Biotechnology 81:813-826.

530 29. Jouhten $\mathrm{P}$, et al. (2016) Yeast metabolic chassis designs for diverse biotechnological $531 \quad$ products. Scientific reports 6. 
532 30. Price ND, Papin JA, Schilling CH, \& Palsson BO (2003) Genome-scale microbial in silico

533 models: the constraints-based approach. Trends in Biotechnology 21:162-169.

534 31. Palsson $\mathrm{B} \varnothing$ (2015) Systems biology: constraint-based reconstruction and analysis.

535 (Cambridge University Press).

536 32. Tepper N \& Shlomi T (2010) Predicting metabolic engineering knockout strategies for 537 chemical production: accounting for competing pathways. Bioinformatics 26:536-543.

538 33. Feist AM, et al. (2010) Model-driven evaluation of the production potential for growth-

540 34. Yang L, Cluett WR, \& Mahadevan R (2011) EMILiO: a fast algorithm for genome-scale $541 \quad$ strain design. Metabolic engineering 13:272-281.

542 35. Fong SS, et al. (2005) In silico design and adaptive evolution of Escherichia coli for 543 production of lactic acid. Biotechnology and bioengineering 91(5):643-648.

544 36. Trinh C \& Srienc F (2009) Metabolic engineering of Escherichia coli for efficient 545 conversion of glycerol to ethanol. Appl Environ Microbiol 75(21):6696 - 6705.

546 37. Coello Coello CA (2002) Theoretical and numerical constraint-handling techniques used 547 with evolutionary algorithms: a survey of the state of the art. Computer Methods in Applied $548 \quad$ Mechanics and Engineering 191(11):1245-1287.

549 38. Marler RT \& Arora JS (2004) Survey of multi-objective optimization methods for $550 \quad$ engineering. Structural and multidisciplinary optimization 26:369-395.

551 39. Deb K, Pratap A, Agarwal S, \& Meyarivan T (2002) A fast and elitist multiobjective 552 genetic algorithm: NSGA-II. Evolutionary Computation, IEEE Transactions on 6:182-197.

553 40. Schellenberger J, et al. (2011) Quantitative prediction of cellular metabolism with 554 constraint-based models: the COBRA Toolbox v2. 0. Nature protocols 6:1290-1307. 
555 41. Heirendt L, et al. (2017) Creation and analysis of biochemical constraint-based models:

556 the COBRA Toolbox v3.0.

557 42. Larhlimi A, David L, Selbig J, \& Bockmayr A (2012) F2C2: a fast tool for the computation 558 of flux coupling in genome-scale metabolic networks. BMC Bioinformatics 13:57.

559 43. Shen CR, et al. (2011) Driving forces enable high-titer anaerobic 1-butanol synthesis in

$560 \quad$ Escherichia coli. Applied and environmental microbiology 77:2905-2915.

561 44. Atsumi S, Hanai T, \& Liao JC (2008) Non-fermentative pathways for synthesis of 562 branched-chain higher alcohols as biofuels. Nature 451:86-89.

563 45. Tseng H-C \& Prather KLJ (2012) Controlled biosynthesis of odd-chain fuels and chemicals 564 via engineered modular metabolic pathways. Proceedings of the National Academy of $565 \quad$ Sciences 109:17925-17930.

566 46. Yim H, et al. (2011) Metabolic engineering of Escherichia coli for direct production of 1, 567 4-butanediol. Nature chemical biology 7:445-452.

568 47. Akita H, Nakashima N, \& Hoshino T (2016) Pyruvate production using engineered 569 Escherichia coli. AMB Express 6(1):94.

570 48. Niu D, et al. (2014) Highly efficient L-lactate production using engineered Escherichia coli with dissimilar temperature optima for L-lactate formation and cell growth. Microbial cell

573 49. Yu JL, Xia XX, Zhong JJ, \& Qian ZG (2014) Direct biosynthesis of adipic acid from a synthetic pathway in recombinant Escherichia coli. Biotechnology and bioengineering

576 50. von Kamp A \& Klamt S (2017) Growth-coupled overproduction is feasible for almost all metabolites in five major production organisms. Nature communications 8:15956. 
578 51. Maranas CD \& Zomorrodi AR (2016) Optimization Methods in Metabolic Networks.

579 52. Hart WE, et al. (2012) Pyomo-optimization modeling in python (Springer).

580 53. Kitano H (2004) Biological robustness. Nature Reviews Genetics 5(11):826.

581 54. Callura JM, Cantor CR, \& Collins JJ (2012) Genetic switchboard for synthetic biology

$582 \quad$ applications. Proceedings of the National Academy of Sciences 109(15):5850-5855.

583 55. Winkler JD, Halweg-Edwards AL, \& Gill RT (2015) The LASER database: Formalizing 584 design rules for metabolic engineering. Metabolic Engineering Communications 2:30-38.

585 56. Coomes MW, Mitchell BK, Beezley A, \& Smith TE (1985) Properties of an Escherichia

586 coli mutant deficient in phosphoenolpyruvate carboxylase catalytic activity. Journal of 587 bacteriology 164(2):646-652.

588 57. Klamt S, Mahadevan R, \& Hädicke O (2018) When Do Two-Stage Processes Outperform $589 \quad$ One-Stage Processes? Biotechnology journal 13(2):1700539. 


\section{$592 \quad$ Figure legends}

593

594 Figure 1: Comparison between the conventional single-product strain design and modular cell

595 engineering. In the conventional approach, each target product requires to go through the iterative

596 optimization cycle. The modular cell engineering approach exploits common phenotypes

597 associated with high product titers, rates, and yields; and hence, the strain optimization cycle only

598 needs to be performed once for multiple products, which helps reduce the cost and time of strain

599 development.

600

601 Figure 2: Graphical representation of phenotypic spaces for different strain design objectives

602 including (A) weak growth coupling $(w G C P)$, (B) strong growth coupling $(s G C P)$, and $(\mathbf{C})$ no-

603 growth production $(N P G) . v_{P k}^{\mu}$ is the minimum product formation rate at the maximum growth

604 rate for production network $k$, and $v_{P_{\max }}^{\mu}$ is the maximum product secretion rate attainable. $v_{P k}^{\bar{\mu}}$

605 and $v_{P_{\text {max }}}^{\bar{\mu}}$ are the minimum and maximum product formation rates for production network $k$

606 during the stationary phase, respectively.

608 Figure 3: Illustration of ModCell2 workflow and analysis including (A) parent model, (B)

609 production modules, $(\mathbf{C})$ design parameters, and (D) simulation output for Pareto set and Pareto

610 front based on design input.

611

612 Figure 4: The 2-D metabolic phenotypic spaces of different $s G C P$ designs using the core

613 metabolic model. (A) Metabolic map, (B) $s G C P-5-0-5$ design, (C) $s G C P-5-0-6$ design, (D) $s G C P$ -

614 5-0-2 design, (E) sGCP-5-1-8 design, (F) $s G C P-6-1-10$ design, and (G) $s G C P-8-2-9$ design. For 
615 each panel, the gray and blue areas correspond to the phenotypic spaces of the wildtype and the

616 optimal production strain, respectively.

618 Figure 5: Comparison of strain design by OptKnock and Modcell2. (A) A correlation between the 619 maximum objective values for each product generated by OptKnock and the equivalent values 620 attained by ModCell2. Each point is colored based on the number of reaction deletions, with 621 warmer colors corresponding to more reaction deletions. (B) A comparison between the Optknock 622 objective vectors with at most 7 reaction deletions and the representative ModCell2 objective 623 vector, $w G C P-7-1$ which dominates them. Each color circle represents a pair of dominating $w G C P$ 624 design and dominated OptKnock solution (Supplementary File S3). (C) Maximum compatibility 625 of OptKnock designs (blue), $w G C P$ designs ( $\beta_{\mathrm{k}}=0$, orange), wGCP designs $\left(\beta_{\mathrm{k}}=1\right.$, yellow). (D) 626 Compatibility distribution of Optknock ( $\alpha=7$, orange) and $w G C P-7-1$ (blue).

628 Figure 6: (A) Pareto front of $w G C P-4-0-d$. The columns correspond to different designs labeled 629 by their design index, $d$, where the rows correspond to different products. (B) Frequency of the top 630 deletion reactions. (C) Product compatibility distribution across designs. (D) Design compatibility. 631 (E) Tradeoff between modularity and performance. The bars correspond to the maximum objective 632 values attainable for each product whereas the blue line represent the objective values of the 633 wGCP-4-0-48alternative design.

635 Figure 7: Production phenotypes of (A) the wild type (gray) and the representative, highly636 compatible design $w G C P-4-0-48$-alternative (blue), (B) the $w G C P \rightarrow N P G$ design, sup-NGP-5-0-

63723 , and $(\mathbf{C})$ the $w G C P \rightarrow s G C P$ design, sup-sGCP-6-0-39. 


\section{$638 \quad$ Figure 1}

\begin{tabular}{|c|c|c|}
\hline Features & $\begin{array}{c}\text { Conventional } \\
\text { strain engineering }\end{array}$ & $\begin{array}{l}\text { Modular cell } \\
\text { engineering }\end{array}$ \\
\hline \multicolumn{3}{|l|}{ Parent strain } \\
\hline Modular cell & Absent & $\begin{array}{l}\text { Optimize } \\
\text { production }\end{array}$ \\
\hline $\begin{array}{l}\text { Exchangeable } \\
\text { modules }\end{array}$ & 1 & Multiple \\
\hline $\begin{array}{l}\text { Optimal } \\
\text { production strains }\end{array}$ & $\widehat{\mathrm{OH}}$ & Module1 $\widehat{O}_{\mathrm{OH}}$ \\
\hline $\begin{array}{l}\text { Design-build-test } \\
\text { cycle }\end{array}$ & $\begin{array}{l}\text { Repeated for every } \\
\text { new product }\end{array}$ & $\begin{array}{l}\text { One time for } \\
\text { many products }\end{array}$ \\
\hline
\end{tabular}

640

641 


\section{Figure 2}

643

644

645
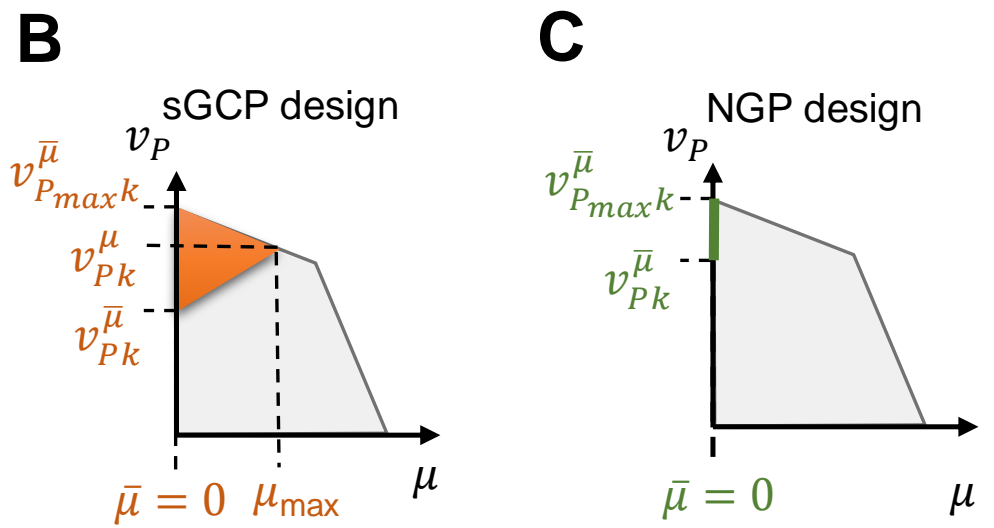

646

647 
A

Parent model

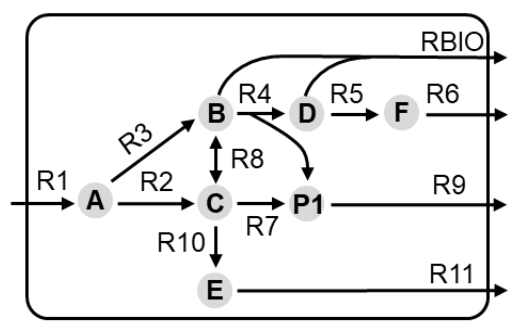

D

Design parameters
B

Production modules

Module 1

Module 2

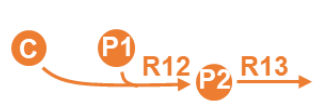

Module 3<smiles>[R16][R6](=O)C(=O)O</smiles>

E
C

\section{Design parameters}

Design objective $\left(f_{k}\right)$

Maximum reaction deletions $(\alpha)$

Maximum module reactions $\left(\beta_{k}\right)$

Pareto front

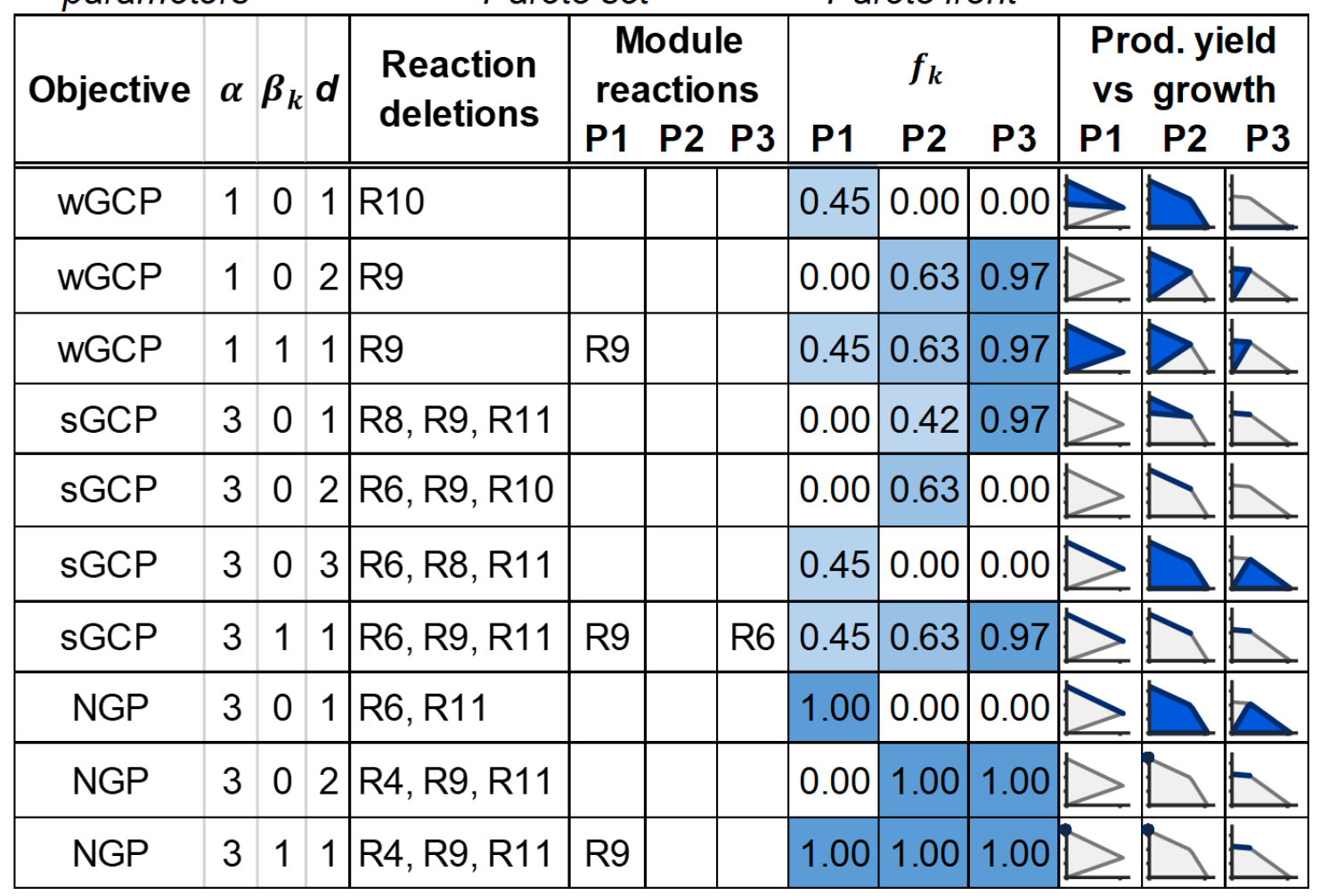

651

652

653 


\section{Figure 4}

A
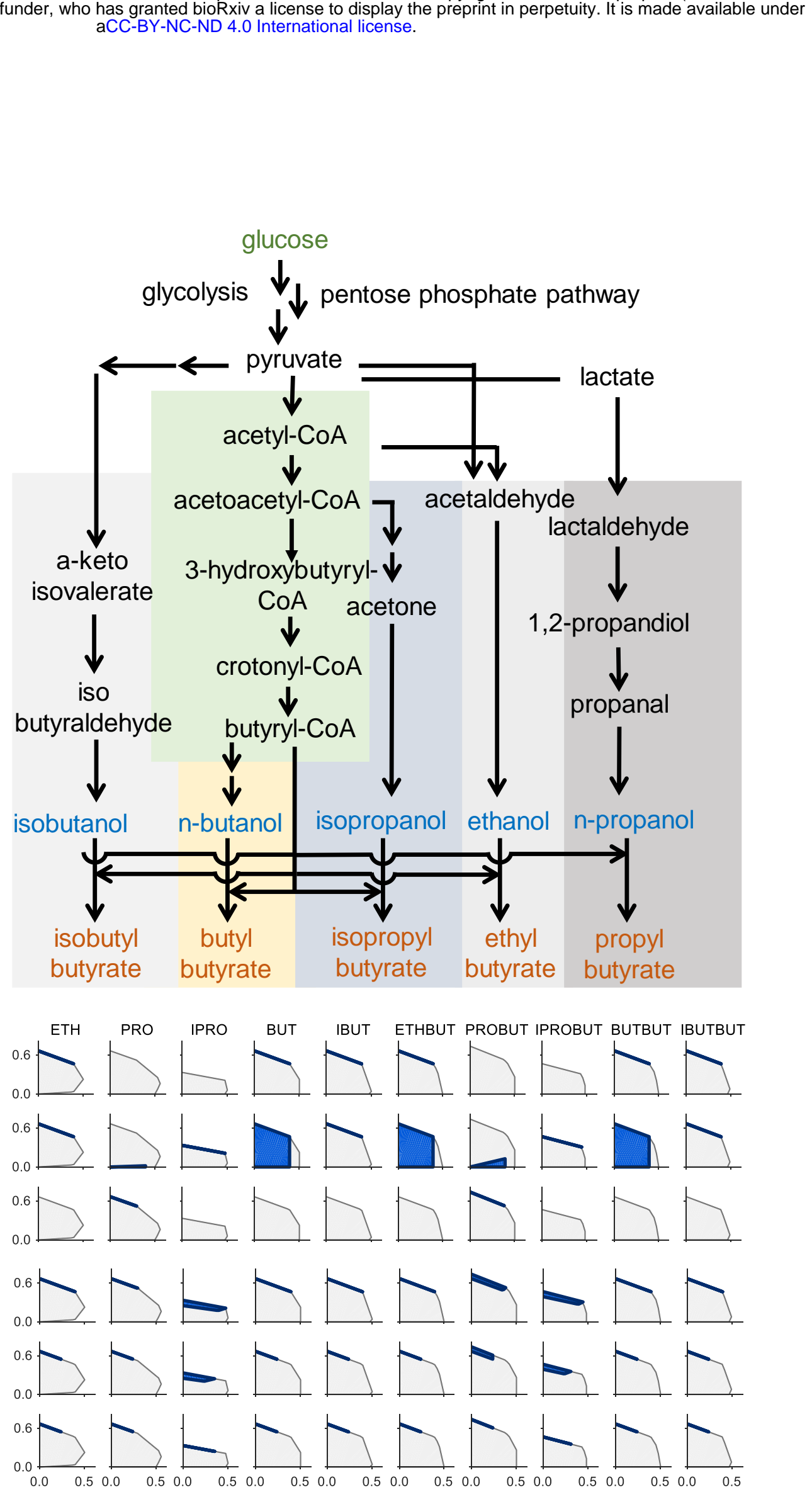

(ticense. preprint in perpetuity. It is made available under
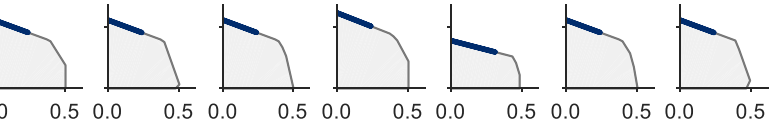

Growth rate $(1 / \mathrm{h})$ 


\section{Figure 5}

657

A

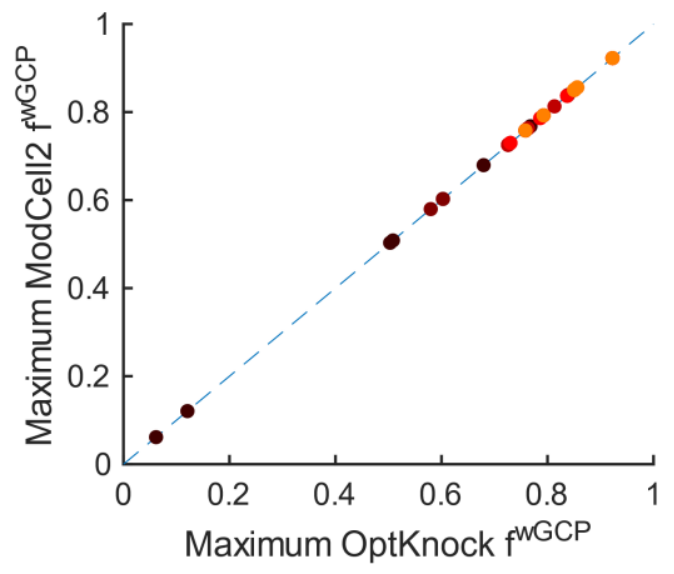

C

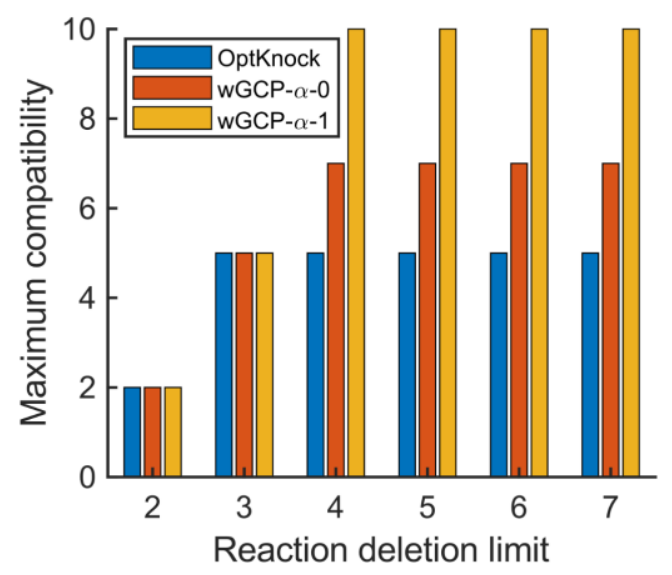

B

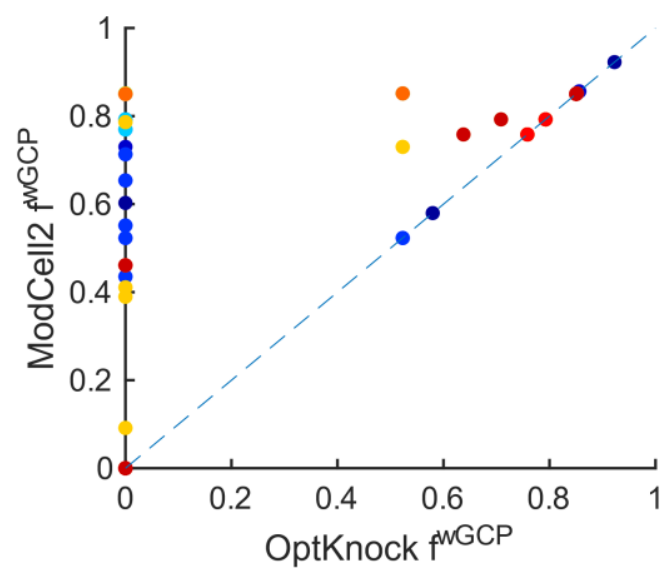

D

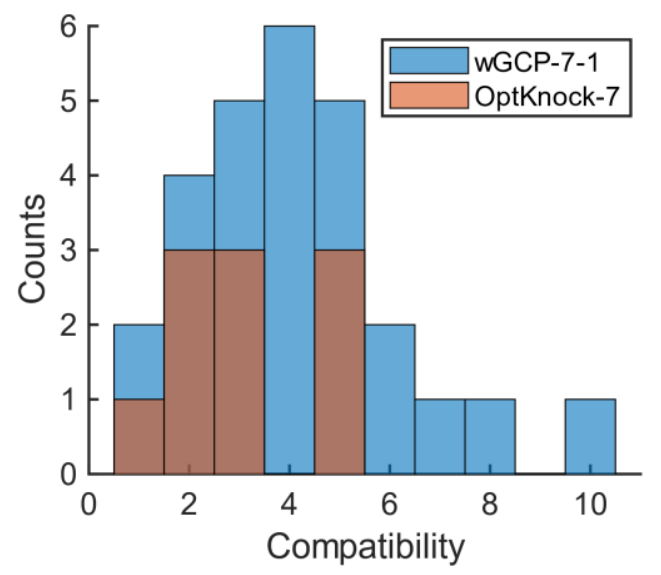


bioRxiv preprint doi: https://doi.org/10.1101/349399; this version posted June 20,2018 . The copyright holder for this preprint (which was not certified by peer review) is the author/funder, who has granted bioRxiv a license to display the preprint in perpetuity. It is made available under aCC-BY-NC-ND 4.0 International license.

\section{Figure 6}

A

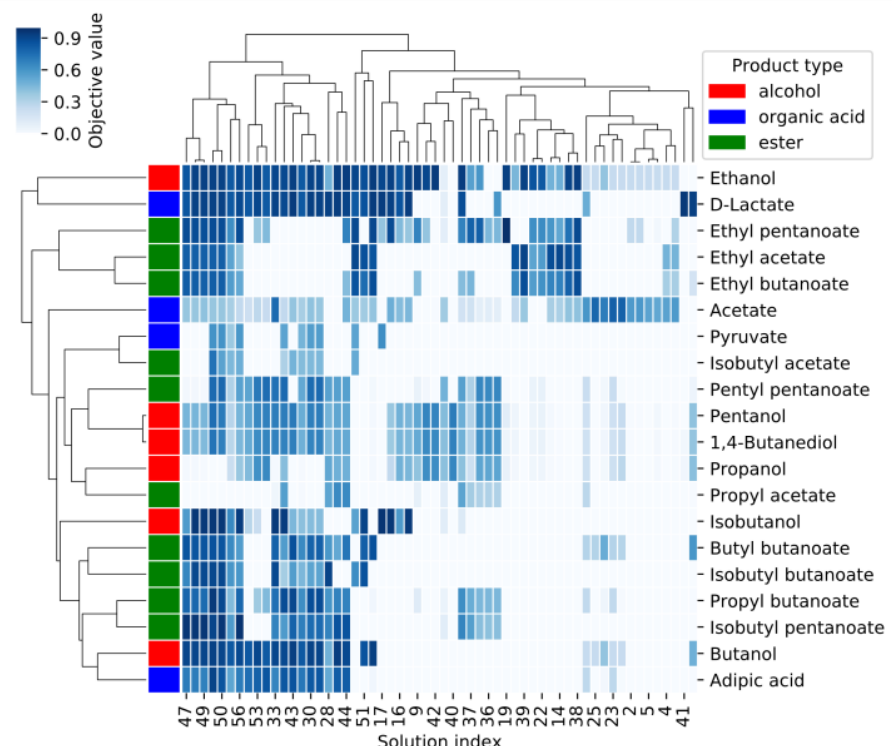

C
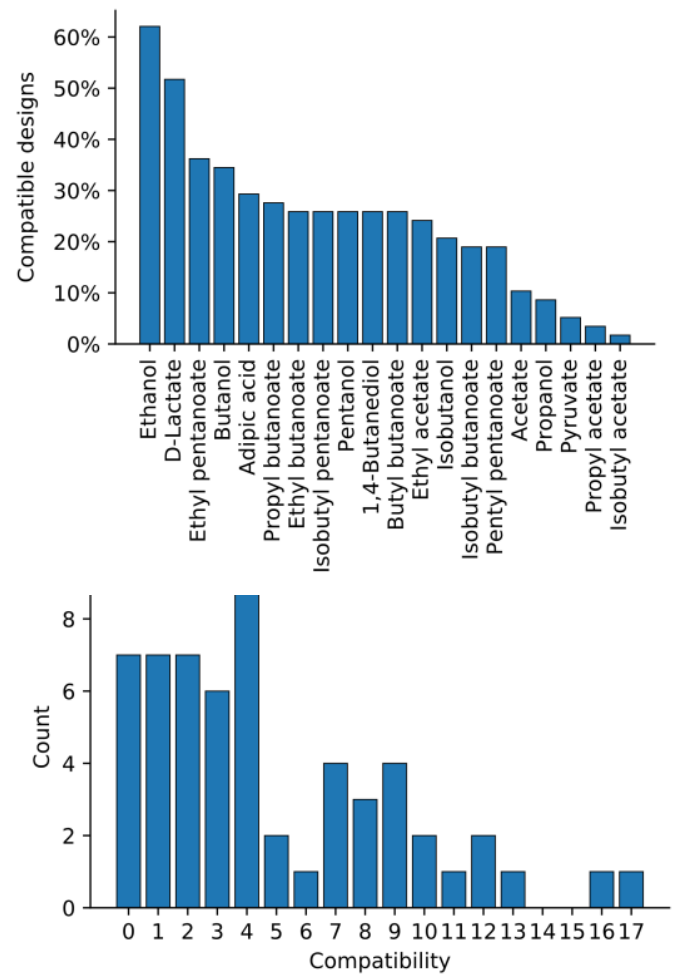

B

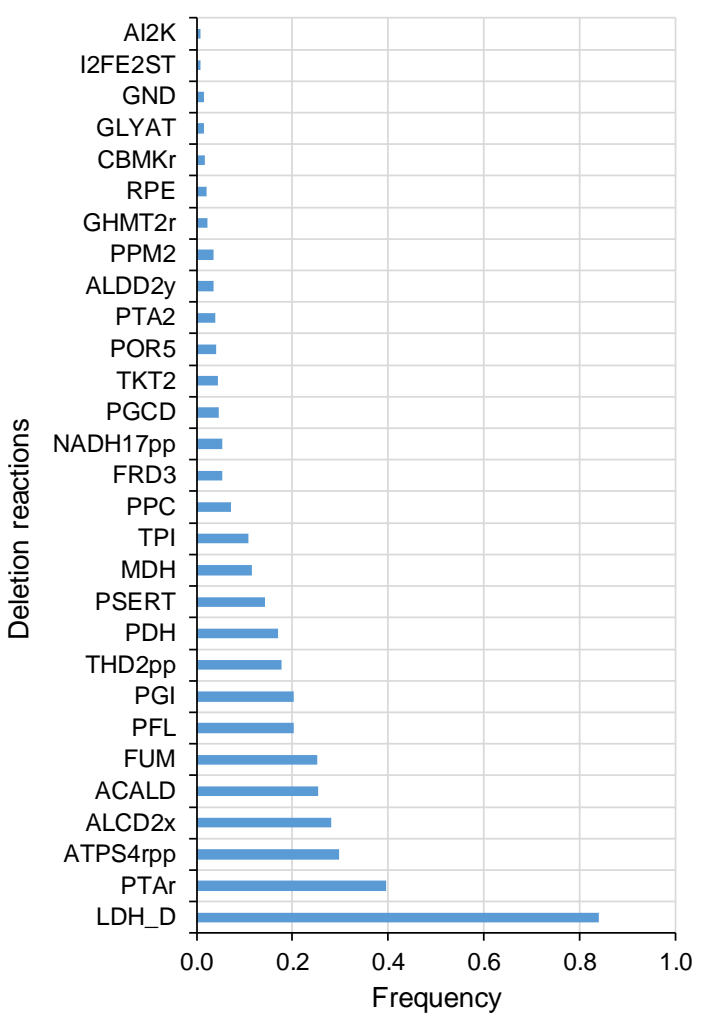

$\mathbf{E}$

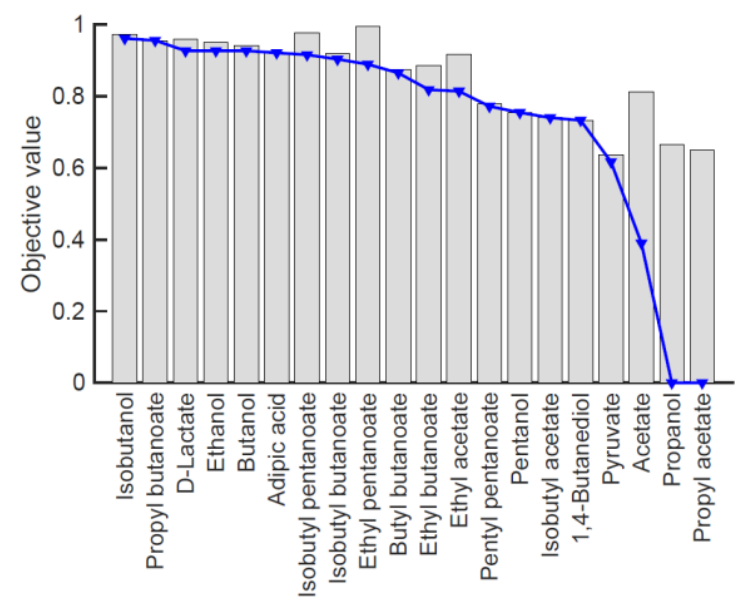

661 


\section{$663 \quad$ Figure 7}

\section{A}

wGCP-4-0-48-design
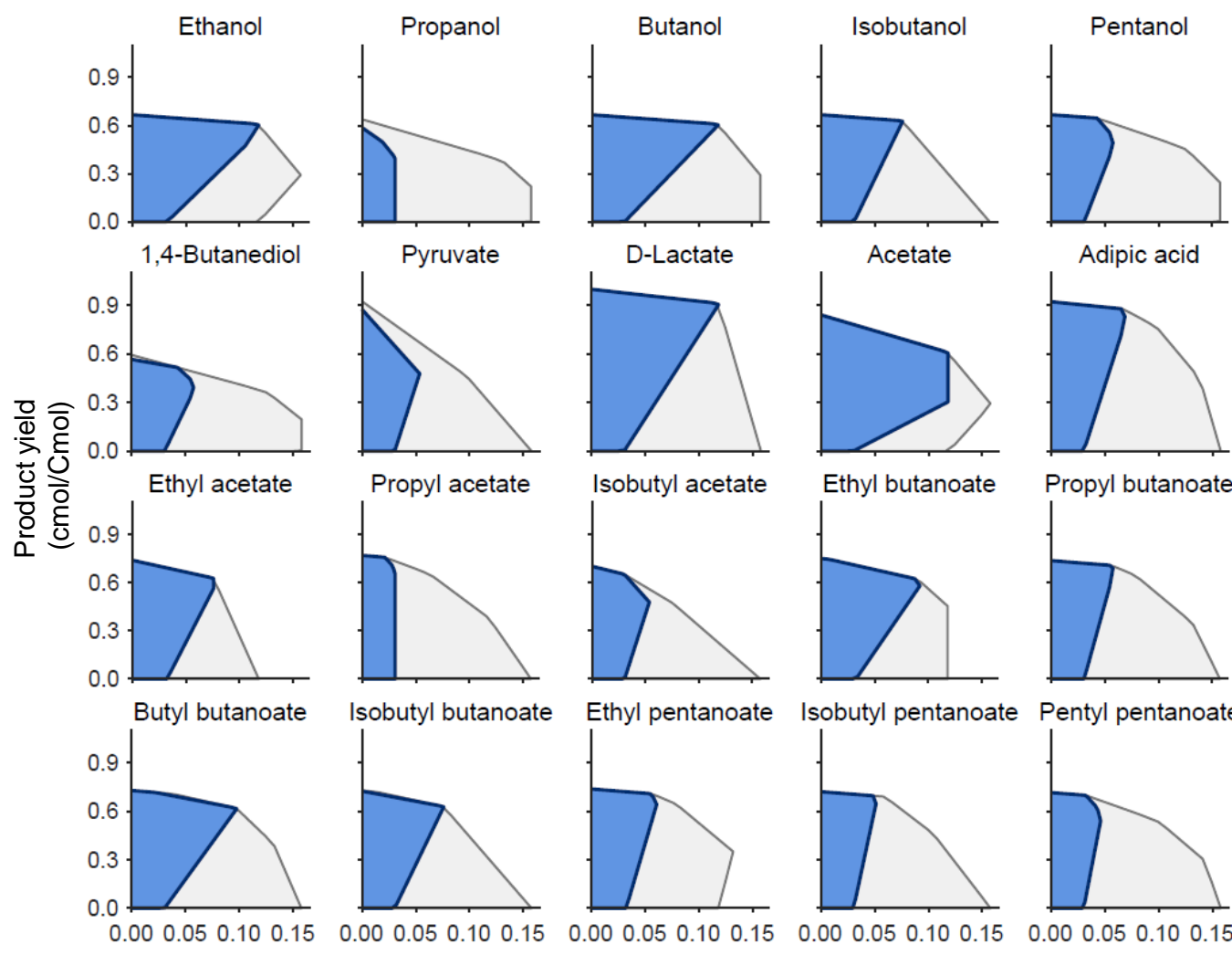

Adipic acid
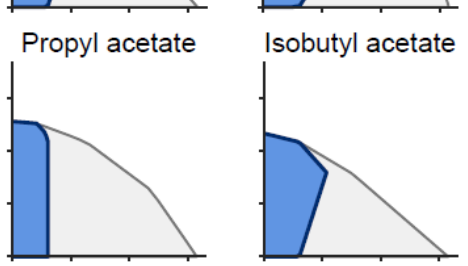

Ethyl butanoate
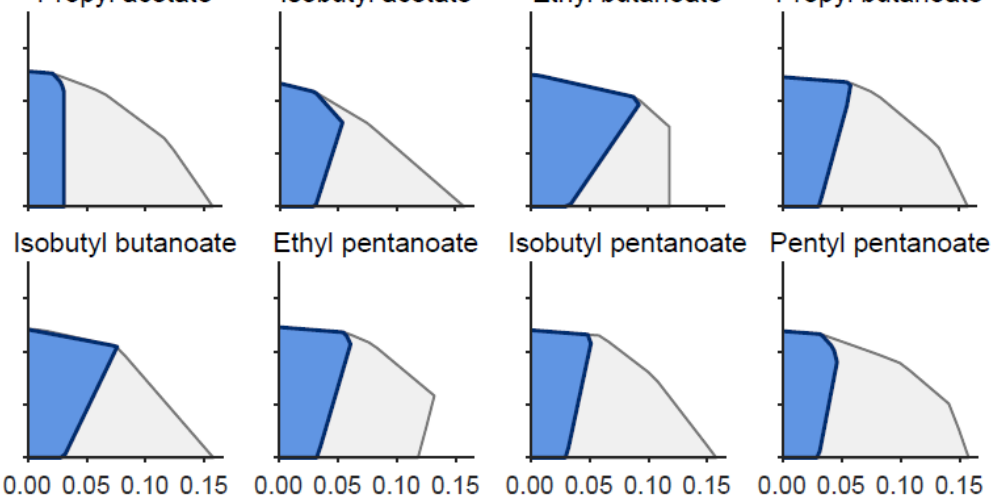

\section{B $w G C P \rightarrow N P G$ design}

Growth rate $(1 / \mathrm{h})$
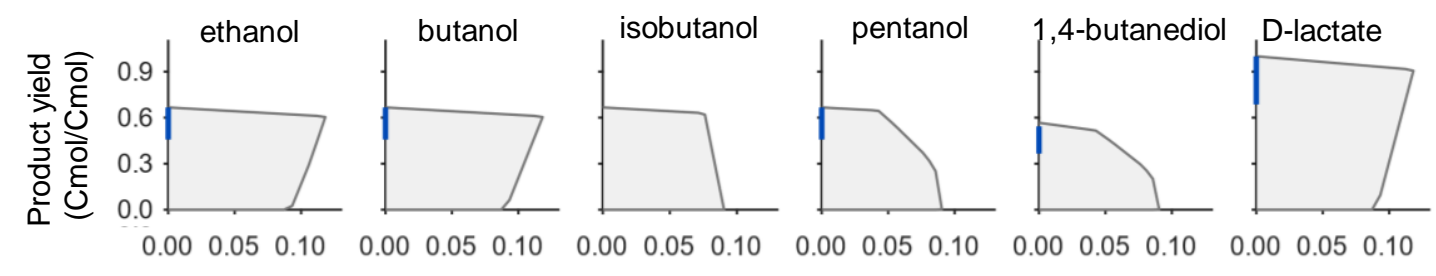

C

$w G C P \rightarrow S G C P$ design

Growth rate (1/h)
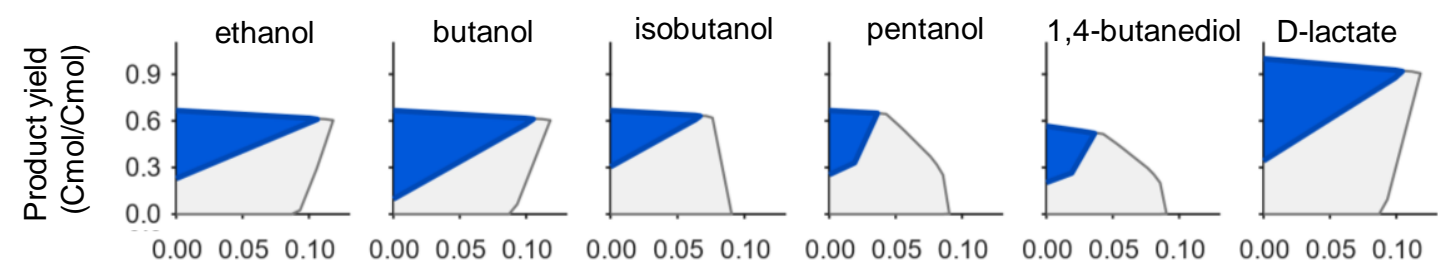

Growth rate (1/h) 